\title{
Lipopolysaccharide expression within the genus Bordetella: influence of temperature and phase variation
}

\author{
Willem M. R. van den Akker \\ Tel: +497071601 718. Fax: +497071610 379.e-mail: willem.akker@tuebingen.mpg.de
}

Max-Planck-Institut für Biologie, Abteilung Infektionsbiologie, Spemannstrasse 34 , D-72076 Tübingen, Germany

\begin{abstract}
LPSs play an important role in bacterial pathogenesis. In this study, the LPS expression of the seven known Bordetella species and its dependency on growth temperature was analysed by oxidative silver staining of proteinase-Ktreated whole bacteria separated by Tricine-SDS-PAGE. The bordetellae were found to have extensively variable LPS in a species-specific way. In addition, the human and ovine Bordetella parapertussis strains exhibited host-specific LPS expression. LPSs from human B. parapertussis strains grown at 37 and $25^{\circ} \mathrm{C}$ were distinct. Growth temperature also affected LPS production by several Bordetella bronchiseptica strains. In some of these cases, BvgAS, the global regulator of virulence factors, was involved in this regulation of LPS biosynthesis. In contrast, no evidence was found for the involvement of the Bordetella pertussis BvgAS system in regulation of LPS synthesis. The obligate human pathogens B. pertussis and Bordetella holmesii are closely related but were shown to produce immunologically distinct LPSs. These species are isolated from the upper respiratory tract and blood, respectively. This raises several interesting questions concerning the potential role of LPS as a virulence factor in the infection processes.
\end{abstract}

Keywords: Bordetella species, BvgAS, lipopolysaccharide expression, phenotypic modulation, phase variation

\section{INTRODUCTION}

The genus Bordetella is composed of a group of Gramnegative bacteria which cause upper respiratory tract infections. The host specificities of bordetellae differ: Bordetella pertussis was isolated from humans, Bordetella parapertussis from humans and sheep, Bordetella bronchiseptica from mammals, Bordetella avium from birds and Bordetella hinzii from birds and humans (Pittman, 1984; Vandamme et al., 1995). Bordetella holmesii was isolated from human blood (Weyant et al., 1995), whereas Bordetella trematum was isolated from wounds and infected ears in humans (Vandamme et al., 1996). The varying host specificities of Bordetella species raises questions about which virulence factors determine host specificity and tissue tropism.

LPSs, as major and essential components of the outermembrane of Gram-negative bacteria, may as such be involved in determining host specificity. The lipid A

Abbreviation: FHA, filamentous haemagglutinin. (endotoxin) part of LPS anchors the glycolipid into the membrane. It is highly conserved and a strong immunogen (Preston et al., 1996). The species-specific functions of LPS are located in the extruding sugar moiety which may contain a long homopolymer of sugar structures (O-antigen) giving rise to smooth LPS (Holst et al., 1996). Such polymeric O-antigens are found in some $B$. parapertussis and B. bronchiseptica strains (Di Fabio et al., 1992). Electrophoretic analysis indicated the presence of two main LPS forms in B.pertussis (the principal causative agent of whooping cough), termed LPSA and the faster migrating LPSB (Peppler, 1984). Structural studies showed that the difference between these LPS forms is the presence of an additional distal trisaccharide in band A (Caroff et al., 1990). mAbs directed against LPSA were able to block bacterial invasion into HeLa 229 cells (Ewanowich et al., 1989). Recently, a gene cluster involved in B. pertussis LPS biosynthesis was identified (Allen \& Maskell, 1996). One of these identified genes ( $w l b G$, previously designated $b p l G$ ) shows sequence homologies with genes required for polysaccharide biosynthesis in other bacteria, and its 
Table 1. Bordetella strains used in this study

\begin{tabular}{|c|c|c|}
\hline Strain & Host/description & Reference/source* \\
\hline \multicolumn{3}{|l|}{ B. pertussis } \\
\hline $\mathrm{BP} 338$ & Human, Tohama I, Nal ${ }^{r}$ & Weiss et al. (1983) \\
\hline BP347 & Tohama I, bvgS:: Tn5 & Weiss et al. (1983) \\
\hline wlbG::kan & Tohama I, wlbG::kan & Allen \& Maskell (1996) \\
\hline BP338ery & Spontaneous $\mathrm{Bvg}^{-}$phase variant of $\mathrm{BP} 338$ & This study \\
\hline B112 & Human, Wellcome $28, \mathrm{Sm}^{\mathrm{r}}$ & Willems et al. (1992) \\
\hline B112ery & Spontaneous $\mathrm{Bvg}^{-}$phase variant of $\mathrm{B} 112$ & This study \\
\hline $18-323$ & Human, strain used for testing vaccines & Pittman (1984) \\
\hline \multicolumn{3}{|l|}{ B. holmesii } \\
\hline 104394 & Human, clinical isolate & CIP \\
\hline 104395 & Human, clinical isolate & CIP \\
\hline \multicolumn{3}{|c|}{ B. parapertussis } \\
\hline $\mathrm{B} 24$ & Human, clinical isolate, The Netherlands & Mooi et al. (1987) \\
\hline MS30 & Human, clinical isolate, Finland & Mertsola (1985) \\
\hline SC7 & Sheep, clinical isolate, Scotland & J. F. Porter, Edinburgh, UK \\
\hline SC10 & Sheep, clinical isolate, Scotland & J. F. Porter, Edinburgh, UK \\
\hline \multicolumn{3}{|c|}{ B. bronchiseptica } \\
\hline B14 & Pig, clinical isolate & van der Zee et al. (1993) \\
\hline B14Bvg- & Spontaneous $\mathrm{Bvg}^{-}$phase variant of B14 & van den Akker (1997) \\
\hline B15 & Pig, clinical isolate & van der Zee et al. (1993) \\
\hline B16 & Pig, clinical isolate & van der Zee et al. (1993) \\
\hline B19 & Dog, clinical isolate & van der Zee et al. (1993) \\
\hline${\mathrm{B} 19 \mathrm{Bvg}^{-}}^{-}$ & Spontaneous $\mathrm{Bvg}^{-}$phase variant of B19 & van den Akker (1997) \\
\hline $\mathrm{B} 20$ & Dog, clinical isolate & van der Zee et al. (1993) \\
\hline B20Bvg- & Spontaneous $\mathrm{Bvg}^{-}$phase variant of $\mathrm{B} 20$ & van den Akker (1997) \\
\hline B81 & Dog, clinical isolate & van der Zee et al. (1993) \\
\hline B615 & Human, clinical isolate & RIVM \\
\hline B618 & Human, clinical isolate & RIVM \\
\hline \multicolumn{3}{|l|}{ B. avium } \\
\hline $\mathrm{B} 21$ & Bird, clinical isolate & $\begin{array}{l}\text { W. Gaastra, Utrecht, The } \\
\text { Netherlands }\end{array}$ \\
\hline $\mathrm{B} 22$ & Bird, clinical isolate & Mooi et al. (1987) \\
\hline \multicolumn{3}{|l|}{ B. trematum } \\
\hline LMG $13506^{\mathrm{T}}$ & $\begin{array}{l}\text { Human, chronic otitis media, Germany } \\
\text { (original designation 1779) }\end{array}$ & Vandamme et al. (1995) \\
\hline \multicolumn{3}{|l|}{ B. hinzii } \\
\hline LMG 10979 & Chicken with slight tracheitis, Belgium & Vandamme et al. (1995) \\
\hline LMG $13501^{\mathrm{T}}$ & Chicken, trachea, Australia & Vandamme et al. (1995) \\
\hline LMG 1872 & Human, sputum, France & Vandamme et al. (1995) \\
\hline
\end{tabular}

* CIP, Collection of Bacterial Strains of Institut Pasteur, Paris, France; RIVM, National Institute of Public Health and the Environment, Bilthoven, The Netherlands.

inactivation resulted in the disappearance of LPSA and its replacement by LPSB (Allen \& Maskell, 1996).

Some Bordetella species, e.g. B. pertussis and B. bronchiseptica, show phase variation caused by mutations in the bvgAS locus resulting in inactivation of the BvgAS twocomponent sensor transducer system (Stibitz \& Miller, 1994; Akerley \& Miller, 1996). BvgAS enhances in a coordinated way the expression of a number of virulence factors such as fimbriae, filamentous haemagglutinin (FHA) and adenylate cyclase toxin/haemolysin. Inactivation of BvgAS results as expected in non-virulent bacteria. Also, environmental conditions such as lowered temperature $\left(25^{\circ} \mathrm{C}\right)$ and sulphate anions result in inactivation of BvgAS (phenotypic modulation).

This study focuses on the LPS expression of the seven known Bordetella species. They appear to exhibit extensive heterogeneity in LPS expression. As some species are not restricted to one single host, isolates from different hosts were included. In all cases the effect of culture at $25^{\circ} \mathrm{C}$ on the LPS expression was investigated, since this is already known to be an important modulator of the expression of virulence factors. For some $B$. bronchiseptica isolates, LPS expression was subject to phase variation in which BvgAS is involved. 


\section{METHODS}

Bacterial strains and growth conditions. Bordetella strains used in this study are listed in Table 1 . Bacteria were routinely grown on Bordet-Gengou agar (Difco) supplemented with $15 \%(\mathrm{v} / \mathrm{v})$ defibrinated sheep blood (BG agar). Plates were incubated in a humidified atmosphere containing $95 \%(\mathrm{v} / \mathrm{v})$ air and $5 \%(\mathrm{v} / \mathrm{v}) \mathrm{CO}_{2}$ at $37^{\circ} \mathrm{C}$. For the study of LPS expression at different temperatures, the bacteria were cultured in liquid Verwey medium (Verwey et al., 1949). To induce phenotypic modulation, $\mathrm{MgSO}_{4}$ was added to the medium to a final concentration of $50 \mathrm{mM}$.

Preparation of LPS samples. Cultures of $30 \mathrm{ml}$ were grown in $100 \mathrm{ml}$ Erlenmeyer flasks approximately to the late exponential growth phase at 37,25 or $41^{\circ} \mathrm{C}$, shaken at 135 r.p.m. LPS of bordetellae was prepared by proteinase $\mathrm{K}$ treatment of lysed bacterial cells using a modification of the method described by Hitchcock \& Brown (1983). Briefly, the organisms grown in broth were harvested by centrifugation and suspended in PBS $(2.68 \mathrm{mM} \mathrm{KCl}, 1.47 \mathrm{mM}$ $\mathrm{KH}_{2} \mathrm{PO}_{4}, 0.49 \mathrm{mM} \mathrm{MgCl}_{2} .6 \mathrm{H}_{2} \mathrm{O}, 137 \mathrm{mM} \mathrm{NaCl}, 8.03 \mathrm{mM}$ $\mathrm{Na}_{2} \mathrm{HPO}_{4} \cdot 2 \mathrm{H}_{2} \mathrm{O}$ ). Agar-grown bacteria were collected with a sterile cotton swab and suspended in PBS. The bacteria present in $80 \mu \mathrm{l}$ of this suspension with an $\mathrm{OD}_{550}$ of 5.0 were pelleted and then resuspended in $100 \mu \mathrm{l}$ water. Subsequently, an equal volume of $2 \times$ sample buffer $(6 \%, \mathrm{w} / \mathrm{v}, \mathrm{SDS} ; 6 \%$, $\mathrm{v} / \mathrm{v}, 2$-mercaptoethanol; $10 \mathrm{mM}$ DTT; $46 \%$, v/v, glycerol; $60 \mathrm{mM}$ Tris, $\mathrm{pH} 8 \cdot 0$; and $0 \cdot 1 \%, \mathrm{w} / \mathrm{v}$, bromophenol blue) was added. After incubation of the samples at $100^{\circ} \mathrm{C}$ for $10 \mathrm{~min}$, the bacterial proteins were digested by adding proteinase $\mathrm{K}$ (Merck) to a final concentration of $50 \mu \mathrm{g} \mathrm{ml}^{-1}$ and incubated at $55^{\circ} \mathrm{C}$ for at least $6 \mathrm{~h}$. The samples were stored at $-20^{\circ} \mathrm{C}$ until analysis by Tricine-SDS-PAGE.

Analysis of LPS by Tricine-SDSPAGE. Electrophoretic profiles of LPS were made by Tricine-SDS-PAGE essentially as described by Lesse et al. (1990). The separating gel of approximately $12 \mathrm{~cm}$ length was prepared with final acrylamide concentrations of $16.4 \% \mathrm{~T}, 6 \% \mathrm{C}$ where $\mathrm{T}$ represents the total percentage $(\mathrm{w} / \mathrm{v})$ of acrylamide (both acrylamide and bisacrylamide) and $C$ represents the percentage of bisacrylamide to the total concentration of acrylamide. The stacking gel of about $1 \mathrm{~cm}$ length consisted of $3.9 \% \mathrm{~T}, 3 \% \mathrm{C}$. The gel was run at a constant current of $20 \mathrm{~mA}$ for about $17 \mathrm{~h}$ at $20^{\circ} \mathrm{C}$. After electrophoresis, the gel was fixed for $24 \mathrm{~h}$ in $40 \%$ $(\mathrm{v} / \mathrm{v})$ ethanol and $5 \%(\mathrm{v} / \mathrm{v})$ acetic acid in water. Bands were visualized by LPS silver staining (Tsai \& Frasch, 1982). The following volumes of LPS samples were added per lane: B. parapertussis, $10 \mu \mathrm{l} ;$ B. avium and B. trematum, $7.5 \mu \mathrm{l}$; and $B$. pertussis, B. holmesii, B. bronchiseptica and B. hinzii, $5 \mu$ l. Partial bromide cleavage products of sperm whale myoglobin (17201, 14632, 8235, 6383 and $2556 \mathrm{Da}$ ) were used as migration markers (Pharmacia Biotech).

Western blot analysis. To determine the reactivity of mAbs raised against Bordetella LPS, proteinase $\mathrm{K}$ digests of whole bacteria were separated on Tricine-SDS-PAGE as described above and directly after electrophoresis blotted onto a nitrocellulose membrane (Protran BA 79 cellulose nitrate, $0.1 \mu \mathrm{m}$; Schleicher \& Schuell). Blotting was carried out in a semi-dry blot apparatus (transfer buffer: $192 \mathrm{mM}$ glycine, $25 \mathrm{mM}$ Tris $/ \mathrm{HCl}$ and $20 \%, \mathrm{v} / \mathrm{v}$, methanol, $\mathrm{pH} 8.3$ ) at $1.5 \mathrm{~mA} \mathrm{~cm}^{-2}$ for $1.5 \mathrm{~h}$. Subsequently, the membrane was washed for $10 \mathrm{~min}$ in Tris-buffered saline with Tween 20 (TBS-T: $20 \mathrm{mM}$ Tris, $500 \mathrm{mM} \mathrm{NaCl}, 0.05 \%$ Tween 20, $\mathrm{pH} 7.5$ ) and blocked for at least $2 \mathrm{~h}$ in TBS-T containing $5 \%$ $(\mathrm{w} / \mathrm{v})$ fat-free milk powder (blocking buffer). After washing three times for $10 \mathrm{~min}$ in TBS-T, the membrane was incubated with a Bordetella LPS mAb diluted in blocking buffer for $4 \mathrm{~h}$. Following another washing procedure, the membrane was incubated for $1.5 \mathrm{~h}$ in blocking buffer containing secondary goat anti-mouse antibodies conjugated with peroxidase (Bio$\mathrm{Rad})$ at a dilution of $1: 2500$. After washing, detection was carried out by enhanced chemiluminescence (ECL, Amersham) according to the manufacturer's instructions.

For the detection of FHA expression, whole-bacterial cell lysates were separated by SDS-PAGE (Laemmli, 1970) in an $8.2 \% \mathrm{~T}, 2.6 \% \mathrm{C}$ separating gel using a minigel system apparatus (Bio-Rad). Blotting on nitrocellulose was done for $2 \mathrm{~h}$ at $2 \mathrm{~mA} \mathrm{~cm}^{-2}$. The next procedure was carried out similarly to that described for the LPS Western blots.

mAbs. The mAbs raised against $B$. pertussis LPS [40A1 (IgG3), 36G3 (IgG1) and 145F12 (IgG3)] and B. pertussis FHA [31E2 (IgG1)] have been described previously by Poolman et al. (1990).

\section{RESULTS}

\section{Increased expression of LPSB in B. pertussis at $25^{\circ} \mathrm{C}$ is not mediated by BvgAS}

$B$. pertussis is an obligate human pathogen colonizing the upper respiratory tract and thereby causing the contagious disease whooping cough (Relman, 1995). The three $B$. pertussis strains studied were haemolytic on $\mathrm{BG}$ agar, indicating that the bacteria were in the $\mathrm{Bvg}^{+}$ state. At $37^{\circ} \mathrm{C}$, the B. pertussis strains expressed a strongly stained LPSA band and a faster-migrating, minor, LPSB band (Fig. 1, sets $1-3$ ). LPSB co-migrates with the LPS of a $w$ lbG mutant (Fig. 2a) which has been shown to express LPSB only (Allen \& Maskell, 1996). Additionally, LPSA appeared to consist of two LPS forms which could be clearly distinguished by TricineSDS-PAGE using a $20 \% \mathrm{~T}, 6 \% \mathrm{C}$ separation gel (data not shown). The slower-migrating LPS form of LPSA was more abundant than the faster-migrating one. Culture at $25^{\circ} \mathrm{C}$ resulted in an increased level of LPSB.

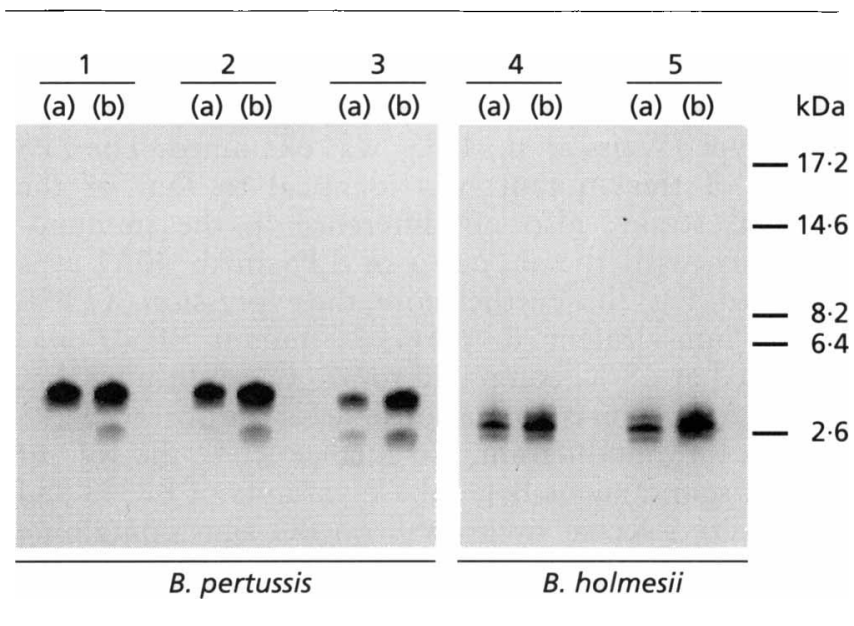

Fig. 1. Electrophoretic profiles of LPS from $B$. pertussis and $B$. holmesii. Strains were cultured at $37^{\circ} \mathrm{C}$ (a) or $25^{\circ} \mathrm{C}$ (b). Sets: 1 , BP338; 2, B112; 3, 18-323; 4, 104394; 5, 104395. Molecular mass markers are included to make comparison of the different gels possible. 
(a)

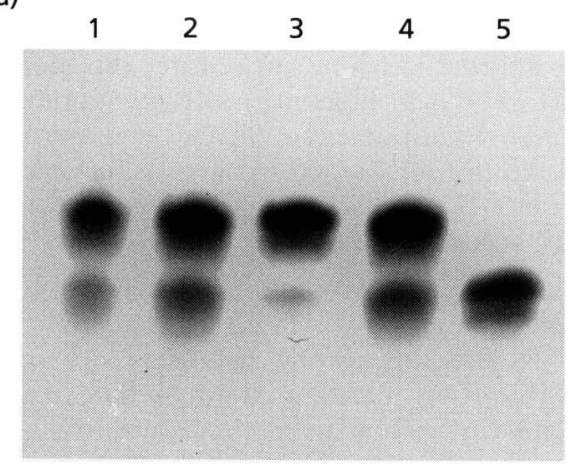

(b)

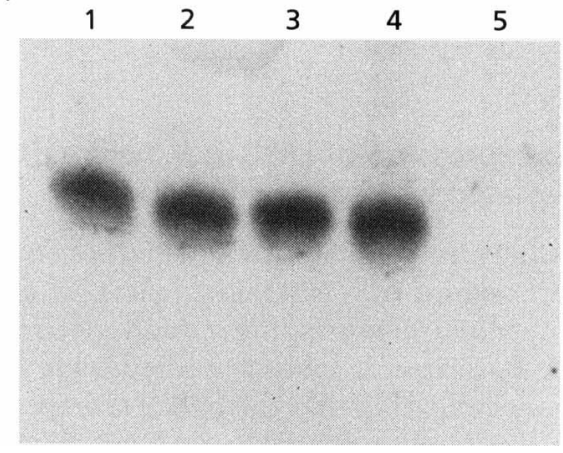

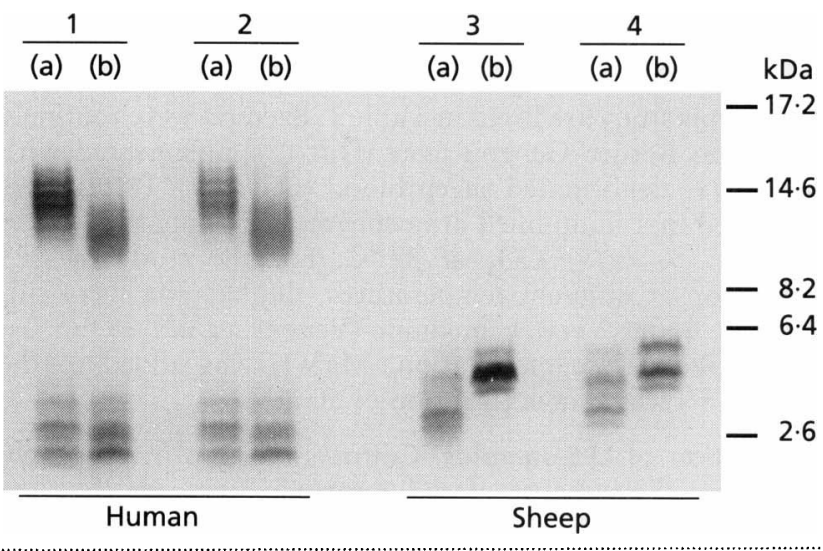

Fig. 3. Electrophoretic profiles of LPS from human and ovine $B$. parapertussis. Strains were cultured at $37^{\circ} \mathrm{C}$ (a) or $25^{\circ} \mathrm{C}$ (b). Sets: 1, B24 (human); 2, MS30 (human); 3, SC7 (sheep); 4, SC10 (sheep).

\section{The human pathogen $B$. holmesii is closely related to B. pertussis but expresses a distinct LPS}

Recently, a novel Bordetella species (B. holmesii) was isolated from the blood of immunocompromised patients (Weyant et al., 1995; Lindquist et al., 1995). The two B. holmesii strains investigated here were nonhaemolytic on BG agar. The LPS profiles of both $B$. holmesii strains were similar and were not modulated by temperature (Fig. 1, sets 4 and 5). Three LPS bands could be distinguished: one major band and two minor ones migrating closely together. None of these bands comigrated with the B. pertussis LPS bands. Western blot analysis demonstrated that the LPS of $B$. holmesii strain 104394 was not recognized by mAbs 40A1, 36G3 and $145 \mathrm{~F} 12$, indicating that $B$. holmesii LPS is distinct from the LPS found in B. pertussis (data not shown). The two $B$. holmesii strains exhibited a homogeneous colony morphology and screening of five randomly chosen colonies of strain 104394 did not provide evidence for intra-strain LPS variation. To investigate whether the BvgAS sensory system is The biosynthesis of B. pertussis LPS and possibly responsible for the temperature-dependent LPSB expression, a Tohama I derivative (BP347) carrying a transposon insertion into bvg $S$ resulting in a $\mathrm{Bvg}^{-}$ phenotype (Weiss et al., 1983) was examined. The LPS profile of this mutant was identical to that of the parental strain; also no difference in the immunoreactivity with the $B$. pertussis LPS $\mathrm{mAb} 40 \mathrm{~A} 1$ was observed (Fig. 2b). Furthermore, the expression of LPSB in the non-virulent $B$. pertussis mutant BP347 was increased at $25^{\circ} \mathrm{C}$ compared to $37^{\circ} \mathrm{C}$, confirming that the BvgAS system is not responsible for the observed LPS temperature modulation. To further study the role of BvgAS, spontaneous Bvg phase variants of BP338 and B112 were selected by growth on BG agar containing erythromycin $\left(0 \cdot 25 \mu \mathrm{g} \mathrm{m}^{-1}\right)$ (Weiss \& Falkow, 1984). These phase variants showed no haemolytic activity and did not differ in LPS expression from their $\mathrm{Bvg}^{+}$parental strains (data not shown). Taken together, the data presented show that BvgAS is not responsible for the temperature-dependent expression level of LPSB and not needed for $B$. pertussis LPS biosynthesis.

\section{Host-specific LPS expression profiles of human and ovine B. parapertussis strains: modulation by temperature}

B. parapertussis has been isolated from both humans and sheep (Linnemann \& Perry, 1977; Cullinane et al., 1987; Porter et al., 1994). The strains studied here differed in their haemolytic activity: the human $B$. parapertussis strains (B24 and MS30) were strongly haemolytic on BG agar whereas the ovine strains (SC7 and SC10) were weakly haemolytic. There are profound differences in the LPS profiles of bacteria isolated from the two hosts (Fig. 3). At $37^{\circ} \mathrm{C}$, the LPS of human $B$. parapertussis strains form a ladder-like pattern in the gel which is characteristic of LPS containing polymeric Oantigens. Interestingly, this pattern is absent at $25^{\circ} \mathrm{C}$ and is instead replaced by a moderately faster-migrating $\mathrm{O}$-antigen containing a single diffuse band. Three fastermigrating major LPS bands can be distinguished in the 


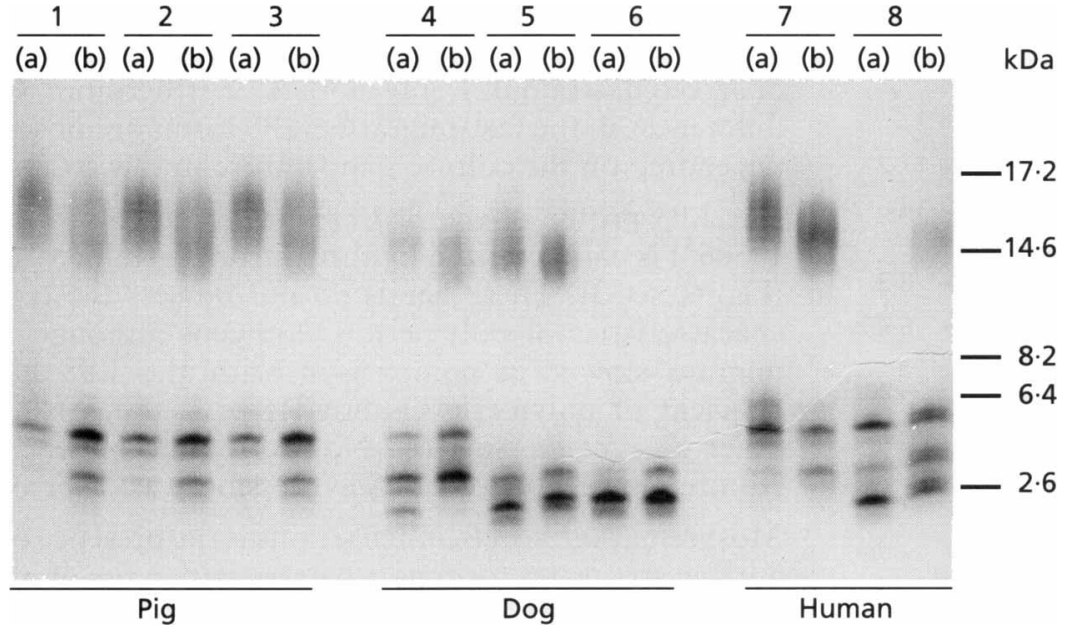

Fig. 4. Electrophoretic profiles of LPS from $B$. bronchiseptica strains from pigs, dogs and humans. Strains were cultured at $37^{\circ} \mathrm{C}$ (a) or $25^{\circ} \mathrm{C}$ (b). Sets: 1, B14 (pig); 2, B15 (pig); 3 , B16 (pig); 4, B19 (dog); 5, B20 (dog); 6, B81 (dog); 7, B615 (human); 8, B618 (human). human strains, and culture at $25^{\circ} \mathrm{C}$ has only minimal effects on their expression. No intra-strain LPS variation was detected by screening of at least 10 colonies of strain B24.

The LPS of the two ovine $B$. parapertussis strains was distinct from that of the human strains, and the LPS biosynthesis is clearly influenced by growth temperature (Fig. 3, sets 3 and 4 ). At $37^{\circ} \mathrm{C}$, two LPS bands are most prominent whereas culture at $25^{\circ} \mathrm{C}$ induces a shift to three more slowly migrating LPS bands. The same LPS profile and temperature effects were also observed in three B. parapertussis strains isolated from New Zealand sheep (unpublished observations). No highmolecular-mass LPS was observed in any of the ovine B. parapertussis strains.

\section{BvgAS controls LPS expression in some B. bronchiseptica strains}

B. bronchiseptica causes respiratory infections in a broad range of mammals (Goodnow, 1980). Here, strains from three hosts were studied: pig $(n=3)$, dog $(n=3)$ and human $(n=2)$. The porcine strains were haemolytic but the canine and human isolates were not. Similarly to the human B. parapertussis strains (Fig. 3), most $B$. bronchiseptica strains express a ladder-forming smooth LPS at $37^{\circ} \mathrm{C}$ and a faster-migrating diffuse Oantigen containing LPS at $25^{\circ} \mathrm{C}$ (Fig. 4). Interestingly, whereas smooth LPS was absent in the human strain B618 at $37^{\circ} \mathrm{C}$, similar diffuse smooth LPS to that seen in other B. bronchiseptica strains was induced upon culture at $25^{\circ} \mathrm{C}$. The canine strains were more heterogeneous in LPS than were the strains from pigs. O-antigen containing high-molecular-mass LPS was absent in the canine $B$. bronchiseptica strain B81 at both temperatures. In all B. bronchiseptica strains temperature is a modulator of LPS expression. FHA was detected in $B$. bronchiseptica strain $\mathrm{B} 14$ grown at $37^{\circ} \mathrm{C}$, but not at $25^{\circ} \mathrm{C}$, with an anti-FHA mAb (data not shown). This indicates that culture at $25^{\circ} \mathrm{C}$ results in phenotypic modulation. Growth of strains B14, B19 and B20 in the presence of sulphate anions, another modulator of
BvgAS, influenced LPS expression as well (Fig. 5a, lanes 3). Since temperature and $\mathrm{MgSO}_{4}$ also influence the bacteria in ways other than via BvgAS, it is not possible to ascribe all observed effects to this sensor system. Therefore, spontaneous phase variants of the B. bronchiseptica strains B14, B19 and B20 (van den Akker, 1997) were investigated. These phase variants swarmed on BG agar, indicating the presence of flagella, a characteristic of the B. bronchiseptica Bvg- phenotype. Also, the B14 phase variant did not express $\mathrm{FHA}$ at $37^{\circ} \mathrm{C}$, which is also indicative of the $\mathrm{Bvg}^{-}$phenotype. The LPS profiles of the three phase variants were clearly different from those of their parental strains and had several characteristics in common with the sulphate-modulated parental strains (Fig. 5a). The experiments depicted in Fig. 5 (a) were repeated with progeny of independently isolated colonies with the $\mathrm{Bvg}^{+}$and $\mathrm{Bvg}^{-}$phenotype and displayed the same LPS profiles as shown in this figure. Some LPS molecules of strains B14 and B19 were recognized by the mAbs $145 \mathrm{~F} 12$ and $36 \mathrm{G} 3$, indicating the presence of a common epitope with $B$. pertussis LPS (Fig. 5b), but no reaction with the LPS of strain B20 was detected (not shown). These immunoblots elucidate the relatedness between LPS forms seen in the LPS profiles and confirm the effect of sulphate modulation and phase variation on the LPS expression. Interestingly, the epitope recognized by $\mathrm{mAb} 36 \mathrm{G} 3$ is also present in the smooth LPS of both strains. In conclusion, the data on the B. bronchiseptica strains B14, B19 and B20 demonstrate a role for BvgAS in the LPS biosynthesis in these strains.

\section{LPS expression in B. avium, B. trematum and B. hinzii}

The Bordetella species discussed so far are genetically closely related and can be viewed as a single species (Musser et al., 1986; Müller \& Hildebrandt, 1993). B. avium and related organisms are more distinct from these bordetellae. Both B. avium (Kersters et al., 1984) and B. hinzii (Vandamme et al., 1995) are pathogens of the respiratory tract of birds, where they can cause bordetellosis and tracheitis, respectively. The recently recognized B. trematum was isolated from ear infections 

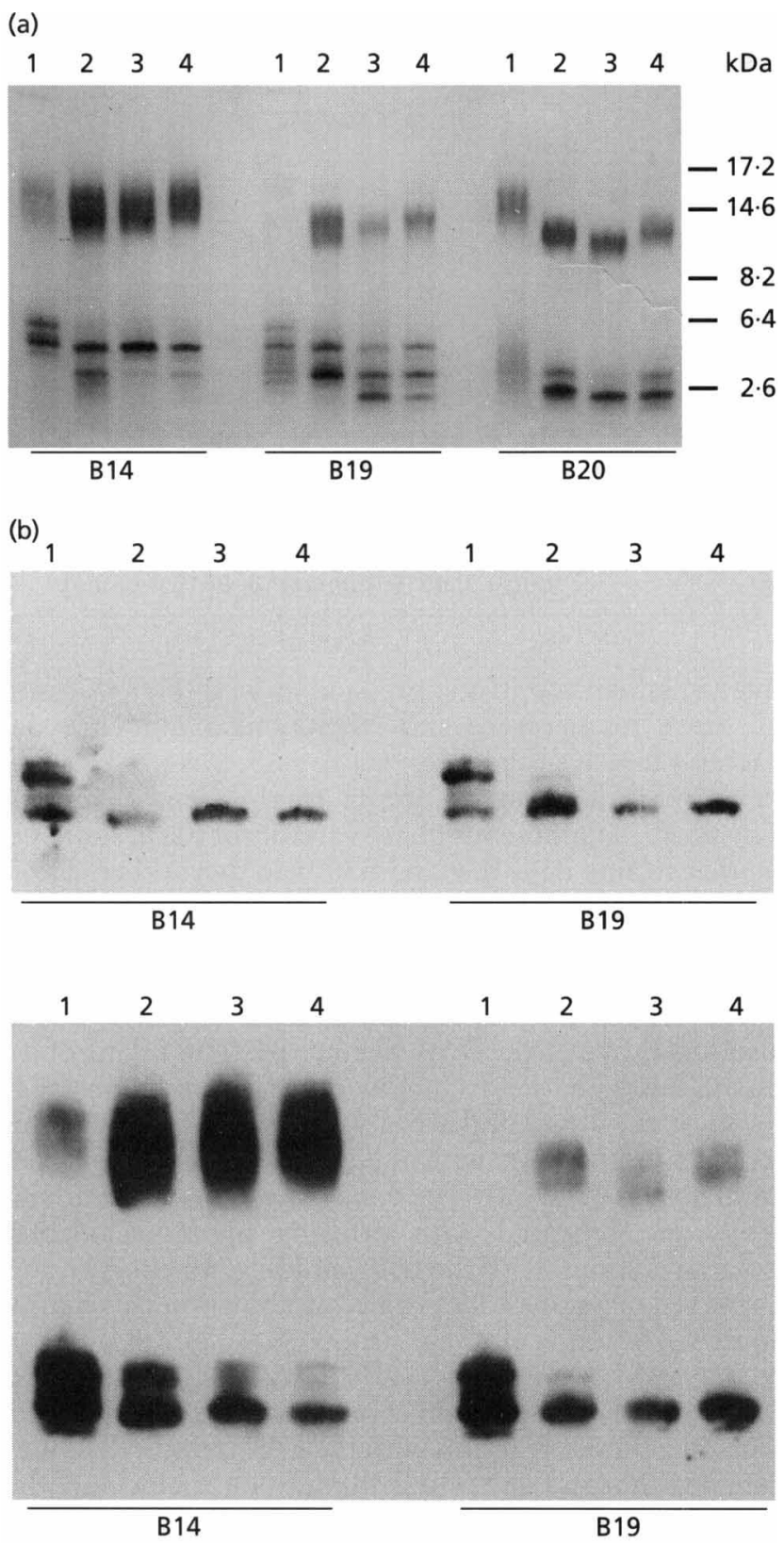

Fig. 5. (a) Effects of temperature, $\mathrm{MgSO}_{4}$ modulation and phase variation on the LPS expression in the $B$. bronchiseptica strains B14, B19 and B20 as analysed by Tricine-SDS-PAGE. Strains were cultured in Verwey broth at $37^{\circ} \mathrm{C}$ (lanes 1), $25^{\circ} \mathrm{C}$ (lanes 2) or supplemented with $50 \mathrm{mM} \mathrm{MgSO}_{4}$ (lanes 3). Lanes 4 contain LPS from the $B$. bronchiseptica phase variants cultured at $37^{\circ} \mathrm{C}$. (b) Western blot of gel (a) developed with mAb 145F12 (upper panel) and mAb $36 \mathrm{G} 3$ (lower panel) raised against $B$. pertussis LPS.

and wounds in humans but its pathogenic significance is unknown (Vandamme et al., 1996). All isolates from the three species are non-haemolytic on BG agar. The two $B$. avium strains have a similar LPS expression at the body temperature of their host $\left(41^{\circ} \mathrm{C}\right)$ but differ in their response to culture at 37 or $25^{\circ} \mathrm{C}$ (Fig. 6 , sets 1 and 2). The LPS profiles of $B$. avium strain B22 are characterized by the constitutive presence of smooth LPS and two fastmigrating LPS bands. This $\mathrm{O}$-antigen containing LPS is observed in strain B21 only at $41^{\circ} \mathrm{C}$. Furthermore, some differences in the fast-migrating LPS forms are observed depending on the culture temperature in this strain.

The LPS profile of the B. trematum type strain (LMG $13506^{\mathrm{T}}$ ) is characterized by three strongly stained bands (Fig. 6, set 3). These bands do not possess the typical characteristics of polymeric $\mathrm{O}$-antigens although they migrate slower in comparison with the LPS bands deficient of polymeric O-antigens as found in several other Bordetella species. No profound influence of culture temperature on LPS expression was detected.

Most remarkable in B. hinzii strains is the presence of $\mathrm{O}$ antigen structures forming a pattern with pairs of bands ('doublets') in the gel (Fig. 7). Although the number of doublets differs from one isolate to another, their migration behaviour is comparable. The expression of this smooth LPS did not depend on the culture temperature, except for a human strain in which the structures were absent at $41^{\circ} \mathrm{C}$. Two fast-migrating LPS forms are observed in all strains independent of the culture temperature. The human $B$. hinzii isolate expresses an additional LPS at $41^{\circ} \mathrm{C}$.

To look for possible (LPS) phase variants, at least 2000 individual colonies were screened with a binocular microscope for variation in colony morphology, but no indications of phase variation were found by this method. In addition, the LPS profiles of at least five randomly selected colonies of the $B$. avium and $B$. trematum strains as well as a human and a chicken (LMG $13501^{\mathrm{T}}$ ) B. hinzii strain did not show LPS variation within a single strain (data not shown). Furthermore, no influence of the presence of $50 \mathrm{mM}$ $\mathrm{MgSO}_{4}$ in BG agar was observed on the LPS profiles of these three species (data not shown).

\section{DISCUSSION}

In this study, Bordetella LPS polymorphism was analysed by Tricine-SDS-PAGE. The data presented suggest species-specific LPS expression; however, the generalization of this notion awaits confirmation by examination of a larger number of strains. Such a study can also answer the question of whether LPS profiles are useful as an additional approach for differentiating bordetellae.

B. pertussis strains exhibit limited LPS variation (rarely a strain lacking LPSA) as was also observed in a study on recent clinical isolates (W. M. R. van den Akker \& F. R. Mooi, unpublished) and is in agreement with other reports (e.g. Peppler, 1984; Chaby \& Caroff, 1988). Based on its co-migration with the LPS of the wlbG mutant in B. pertussis Tohama I (Fig. 2), LPSB seems to be the trisaccharide-deficient LPS form. Interestingly, by use of a higher acrylamide concentration and longer electrophoresis time, it was found that LPSA consists of two LPS forms. This could possibly result from the presence or absence of a phosphate group on a 3-deoxyD-manno-2-octulosonic acid unit in the B. pertussis LPS 


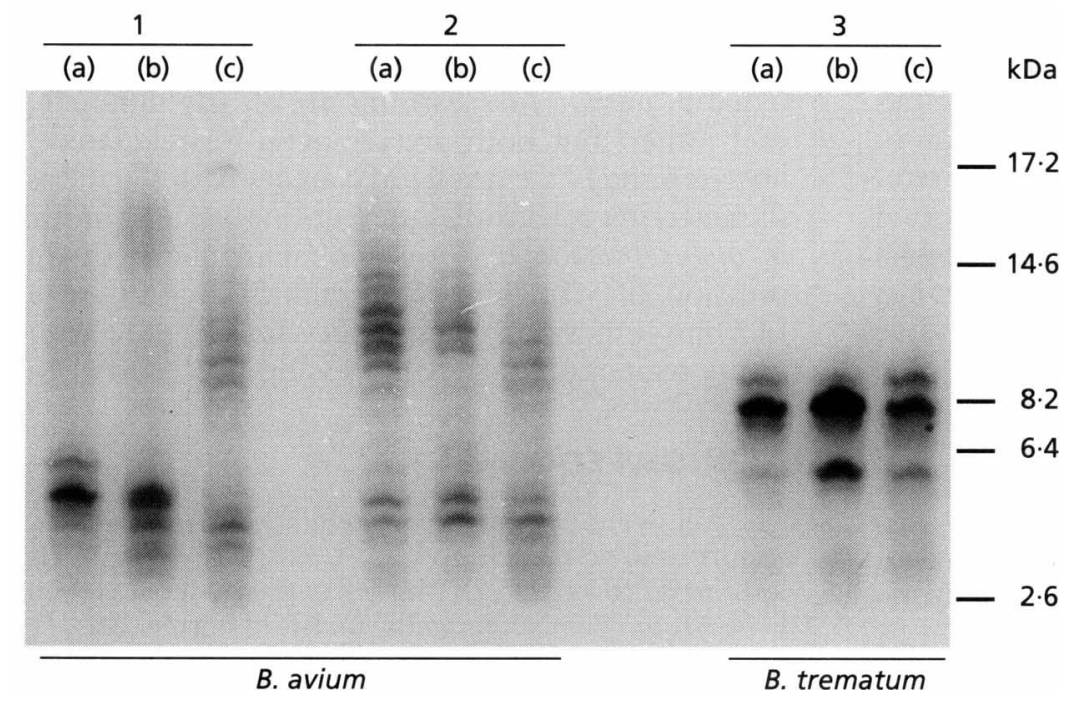

Fig. 6. Electrophoretic profiles of LPS from $B$. avium and $B$. trematum. Strains were cultured at $37^{\circ} \mathrm{C}$ (a), $25^{\circ} \mathrm{C}$ (b) or $41^{\circ} \mathrm{C}$ (c). Sets: 1, B21; 2, B22; 3, LMG $13506^{\top}$.

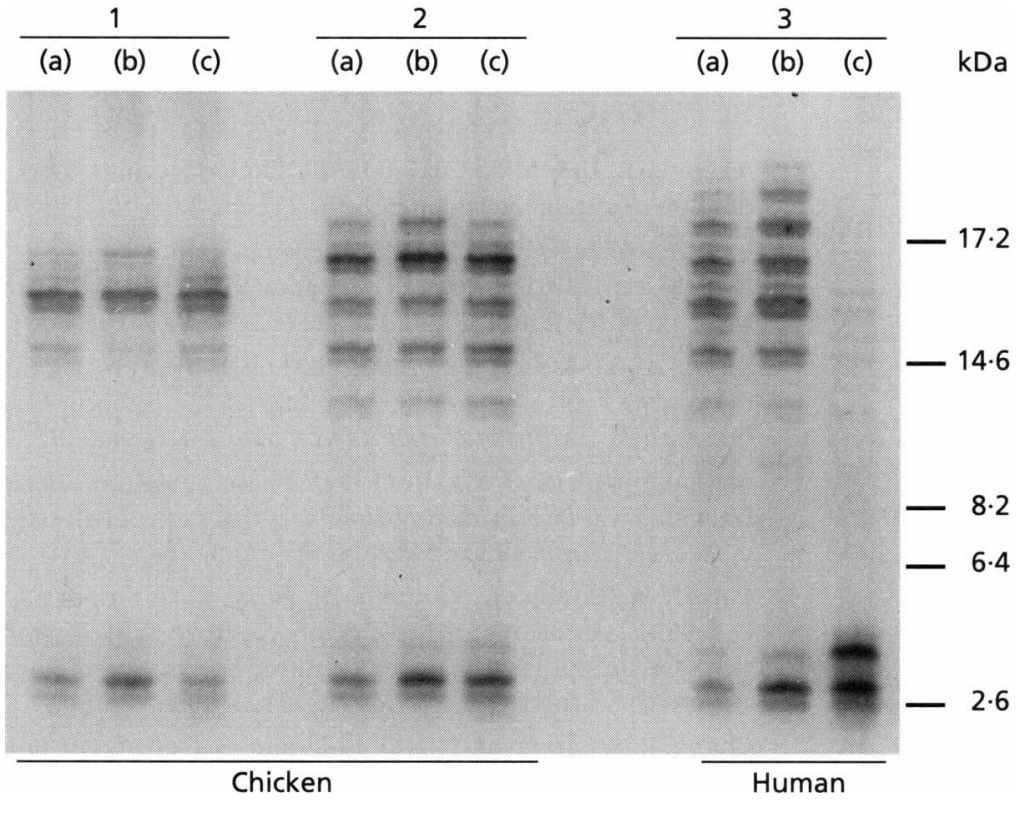

Fig. 7. Electrophoretic profiles of LPS from B. hinzii strains from chickens and humans. Strains were cultured at $37^{\circ} \mathrm{C}$ (a), $25^{\circ} \mathrm{C}$ (b) or $41^{\circ} \mathrm{C}$ (c). Sets: 1, LMG 10979; 2, LMG $13501^{\top} ; 3$, LMG 1872.

(Le Dur et al., 1980), or might result from variability in fatty acid substitution as described for B. bronchiseptica LPS (Zarrouk et al., 1997). Studies of a BvgAS-deficient transposon mutant and spontaneous phase variants suggest that BvgAS is not involved in LPS biosynthesis in $B$. pertussis. B. holmesii is a species closely related to $B$. pertussis (Weyant et al., 1995) but it had a different LPS profile and there was no cross-reactivity of $B$. pertussis LPS mAbs with the LPS of $B$. holmesii.

B. parapertussis exhibits host-specific LPS expression. This was further confirmed by a study of more human and ovine B. parapertussis strains (unpublished observations), and extends previous observations that human and ovine isolates differ in the presence of LPS epitopes (Porter et al., 1995). Combined with differences found in fatty acid expression, antibiotic resistance patterns (Porter et al., 1995) and the presence of insertion sequence elements (van der Zee et al., 1996), this suggests that human and ovine strains form separate lineages. Parallels in LPS structure and regulation were observed between the two human $B$. parapertussis strains and six out of eight $B$. bronchiseptica strains. In particular, the presence and temperature modulation of two polymeric $\mathrm{O}$-antigen structures was remarkable (Figs 3 and 4). One canine strain (B81), possibly a phase variant, did not produce high-molecular-mass LPS and no temperature modulation was observed (Fig. 4, set 6). In the case of a human strain (B618) smooth LPS was only expressed at $25^{\circ} \mathrm{C}$ (Fig. 4, set 8). Despite these exceptions, this LPS phase variation seems to be a common phenomenon among $B$. bronchiseptica strains and was not restricted to a particular host. Cross-reactivity of LPS mAbs (Le Blay et al., 1994) and the presence of a common sugar structure (a homopolymer of 1,4-linked 2,3-diacetamido-2,3-dideoxy- $\alpha$-L-galactopyranosyluronic acid res- 
idues) in the O-antigen (Di Fabio et al., 1992) also stress the relatedness in LPS present in human B. parapertussis and B. bronchiseptica strains. Together these similarities strongly point to a mutual progenitor or acquisition of LPS genes by horizontal transfer. The effects of temperature and sulphate anions on LPS profiles, supported by the phase variants, indicate that the BvgAS system regulates LPS expression in some $B$. bronchiseptica strains. Previously, Peppler \& Schrumpf (1984) also reported differences in LPS profiles between $\mathrm{Bvg}^{+}$and $\mathrm{Bvg}^{-}$B. bronchiseptica phase variants. The B. bronchiseptica strains B14 and B19 appear to have LPS structures in common with B. pertussis LPS as demonstrated by cross-reactivity of mAbs. The already discussed similarities in LPS of human $B$. parapertussis isolates and $B$. bronchiseptica isolates might indicate that BvgAS plays a role in B. parapertussis LPS regulation. In fact, we have evidence that BvgAS is indeed implicated in B. parapertussis LPS expression (W. M. R. van den Akker, T. F. Meyer \& F. R. Mooi, unpublished).

Gentry-Weeks et al. (1991) reported phase variation in a B. avium strain at a frequency of $2 \times 10^{-4}$. These phase variants had a different colony morphology and lacked five outer-membrane proteins. Those five proteins were also absent when the original strain was grown in the presence of $\mathrm{MgSO}_{4}$. Neither phase variants nor an influence of $\mathrm{MgSO}_{4}$ on LPS expression were found in the two $B$. avium strains studied here. The possibility that these strains were phase variants must be acknowledged.

The biological roles of the different LPS molecules found in the Bordetella species are still an enigma. It is, for example, intriguing that the closely related $B$. pertussis and $B$. holmesii species are found in different niches (respiratory tract versus blood) and differ in LPS expression. The dependency of Bordetella LPS expression on environmental conditions, as demonstrated here, exemplifies the dynamics of the expression of virulence determinants in these pathogenic bacteria. In this respect, it is of special interest that $B$. bronchiseptica bvgAS mutants have an advantage in survival over wildtype strains in phagocytes (Banemann \& Gross, 1997), which might hint at a role for LPS in the intracellular environment. Further knowledge about heterogeneity, structure and regulation of LPS combined with information about pathogenesis is essential to be able to understand the functions of the different LPS forms in the dynamic environment that pathogens encounter during infection.

\section{Conclusions}

The main conclusions of this study can be summarized as follows. (1) There is extensive variation among the seven Bordetella species in LPS expression as analysed by electrophoresis. This LPS expression seems to be species-specific and may offer possibilities for determination and differentiation. (2) Temperature is a modulator of LPS expression in most Bordetella species. (3) The LPSs expressed by the closely related B. pertussis and B. holmesii species are phenotypically and immunologically distinct. (4) The LPSs expressed by human and ovine $B$. parapertussis strains are clearly different from each other, but both are temperature regulated. This host-specific LPS expression concurs with the thesis that these strains constitute different lineages. (5) In some $B$. bronchiseptica strains the global regulator of the expression of virulence determinants (BvgAS) regulates LPS biosynthesis. (6) BvgAS does not seem to participate in the regulation of LPS expression in B. pertussis.

\section{ACKNOWLEDGEMENTS}

The following persons are gratefully thanked for providing strains and/or mAbs: Drs W. Gaastra (The Netherlands), K. Kersters (Belgium), D. Maskell (UK), J. Mertsola (Finland), F. R. Mooi (The Netherlands) and J. F. Porter (UK). I thank Frits R. Mooi, Scott D. Gray-Owen and Bernard A. M. van der Zeijst for critical comments on the manuscript. Thomas F. Meyer is thanked for his interest and offering the opportunity to work in his laboratory. Carmen Müller is acknowledged for photography.

\section{REFERENCES}

Akerley, B. J. \& Miller, J. F. (1996). Understanding signal transduction during bacterial infection. Trends Microbiol 4, 141-146. van den Akker, W. M. R. (1997). Bordetella bronchiseptica has a BvgAS-controlled cytotoxic effect upon interaction with epithelial cells. FEMS Microbiol Lett 156, 239-244.

Allen, A. \& Maskell, D. (1996). The identification, cloning and mutagenesis of a genetic locus required for lipopolysaccharide biosynthesis in Bordetella pertussis. Mol Microbiol 19, 37-52.

Banemann, A. \& Gross, R. (1997). Phase variation affects longterm survival of Bordetella bronchiseptica in professional phagocytes. Infect Immun 65, 3469-3473.

Caroff, M., Chaby, R., Karibian, D., Perry, J., Deprun, C. \& Szabó, L. (1990). Variations in the carbohydrate regions of Bordetella pertussis lipopolysaccharides: electrophoretic, serological, and structural features. J Bacteriol 172, 1121-1128.

Chaby, R. \& Caroff, M. (1988). Lipooligosaccharides of Bordetella pertussis endotoxin. In Pathogenesis and Immunity in Pertussis, pp. 247-271. Edited by A. C. Wardlaw \& R. Parton. Chichester: Wiley.

Cullinane, L. C., Alley, M. R., Marshall, R. B. \& Manktelow, B. W. (1987). Bordetella parapertussis from lambs. N Z Vet J 35, 175.

Di Fabio, J. L., Caroff, M., Karibian, D., Richards, J. C. \& Perry, M. B. (1992). Characterization of the common antigenic lipopolysaccharide O-chains produced by Bordetella bronchiseptica and Bordetella parapertussis. FEMS Microbiol Lett 97, 275-282.

Ewanowich, C. A., Melton, A. R., Weiss, A. A., Sherburne, R. K. \& Peppler, M. S. (1989). Invasion of HeLa 229 cells by virulent Bordetella pertussis. Infect Immun 57, 2698-2704.

Gentry-Weeks, C. R., Provence, D. L., Keith, J. M. \& Curtiss, R., III (1991). Isolation and characterization of Bordetella avium phase variants. Infect Immun 59, 4026-4033.

Goodnow, R. A. (1980). Biology of Bordetella bronchiseptica. Microbiol Rev 44, 722-738.

Hitchcock, P. J. \& Brown, T. M. (1983). Morphological heterogeneity among Salmonella lipopolysaccharide chemotypes in silver-stained polyacrylamide gels. J Bacteriol 154, 269-277.

Holst, O., Ulmer, A. J., Brade, H., Flad, H.-D. \& Rietschel, E. T. 
(1996). Biochemistry and cell biology of bacterial endotoxins. FEMS Immunol Med Microbiol 16, 83-104.

Kersters, K., Hinz, K.-H., Hertle, A., Segers, P., Lievens, A., Siegmann, O. \& De Ley, J. (1984). Bordetella avium sp. nov., isolated from the respiratory tracts of turkeys and other birds. Int J Syst Bacteriol 34, 56-70.

Laemmli, U. K. (1970). Cleavage of structural proteins during the assembly of the head of bacteriophage T4. Nature 227, 680-685.

Le Blay, K., Caroff, M., Richards, J. C., Perry, M. B. \& Chaby, R. (1994). Specific and cross-reactive monoclonal antibodies to Bordetella parapertussis and Bordetella bronchiseptica lipopolysaccharides. Microbiology 140, 2459-2465.

Le Dur, A., Chaby, R. \& Szabó, L. (1980). Isolation of two proteinfree and chemically different lipopolysaccharides from Bordetella pertussis phenol-extracted endotoxin. J Bacteriol 143, 78-88.

Lesse, A. J., Campagnari, A. A., Bittner, W. E. \& Apicella, M. A. (1990). Increased resolution of lipopolysaccharides and lipooligosaccharides utilizing tricine-sodium dodecyl sulfate-polyacrylamide gel electrophoresis. J Immunol Methods 126, 109-117.

Lindquist, S. W., Weber, D. J., Mangum, M. E., Hollis, D. G. \& Jordan, J. (1995). Bordetella holmesii sepsis in an asplenic adolescent. Pediatr Infect Dis J 14, 813-815.

Linnemann, C. C. \& Perry, E. B. (1977). Bordetella parapertussis. Recent experience and a review of the literature. Am J Dis Child 131, 560-563.

Mertsola, J. (1985). Mixed outbreak of Bordetella pertussis and Bordetella parapertussis infection in Finland. Eur J Clin Microbiol 4, 123-128.

Mooi, F. R., van der Heide, H. G. J., ter Avest, A. R., Welinder, K. G., Livey, I., van der Zeijst, B. A. M. \& Gaastra, W. (1987). Characterization of fimbrial subunits from Bordetella species. Microb Pathog 12, 127-135.

Müller, M. \& Hildebrandt, A. (1993). Nucleotide sequences of the 23S rRNA genes from Bordetella pertussis, B. parapertussis, $B$. bronchiseptica and $B$. avium, and their implications for phylogenetic analysis. Nucleic Acids Res 21, 3320.

Musser, J. M., Hewlett, E. L., Peppler, M. S. \& Selander, R. K. (1986). Genetic diversity and relationships in populations of Bordetella species. J Bacteriol 166, 230-237.

Peppler, M. S. (1984). Two physically and serologically distinct lipopolysaccharide profiles of Bordetella pertussis and their phenotypic variants. Infect Immun 43, 224-232.

Peppler, M. S. \& Schrumpf, M. E. (1984). Phenotypic variation and modulation in Bordetella bronchiseptica. Infect Immun 44, 681-687.

Pittman, M. (1984). Genus Bordetella Moreno-López 1952, 178 ${ }^{\mathrm{AL}}$. In Bergey's Manual of Systematic Bacteriology, vol. 1, pp. 388-393. Edited by N. R. Krieg \& J. G. Holt. Baltimore: Williams \& Wilkins.

Poolman, J. T., Kuipers, B., Vogel, M. L., Hamstra, H. J. \& Nagel, J. (1990). Description of a hybridoma bank towards Bordetella pertussis toxin and surface antigens. Microb Pathog 8, 377-382.

Porter, J. F., Connor, K. \& Donachie, W. (1994). Isolation and characterization of Bordetella parapertussis-like bacteria from ovine lungs. Microbiology 140, 255-261.

Porter, J. F., Connor, K., van der Zee, A., Reubsaet, F., Ibsen, P., Heron, I., Chaby, R., Le Blay, K. \& Donachie, W. (1995). Characterisation of ovine Bordetella parapertussis isolates by analysis of specific endotoxin (lipopolysaccharide) epitopes, filamentous haemagglutinin production, cellular fatty acid composition and antibiotic sensitivity. FEMS Microbiol Lett 132, 195-201.

Preston, A., Mandrell, R. E., Gibson, B. W. \& Apicella, M. A. (1996). The lipooligosaccharides of pathogenic Gram-negative bacteria. Crit Rev Microbiol 22, 139-180.

Relman, D. A. (1995). Bordetella pertussis: determinants of virulence. In Bacterial Toxins and Virulence Factors in Disease. Handbook of Natural Toxins, vol. 8, pp. 367-405. Edited by J. Moss, B. Iglewski, M. Vaughan \& A. T. Tu. New York: Marcel Dekker.

Stibitz, S. \& Miller, J. F. (1994). Coordinate regulation of virulence in Bordetella pertussis mediated by the vir (bvg) locus. In Molecular Genetics of Bacterial Pathogenesis, pp. 407-422. Edited by V. L. Miller, J. B. Kaper, D. A. Portnoy \& R. R. Isberg. Washington, DC: American Society for Microbiology.

Tsai, C.-M. \& Frasch, C.E. (1982). A sensitive silver stain for detecting lipopolysaccharides in polyacrylamide gels. Anal Biochem 119, 115-119.

Vandamme, P., Hommez, J., Vancanneyt, M., Monsieurs, M., Hoste, B., Cookson, B., Wirsing von König, C. H., Kersters, K. \& Blackall, P. J. (1995). Bordetella hinzii sp. nov., isolated from poultry and humans. Int J Syst Bacteriol 45, 37-45.

Vandamme, P., Heyndrickx, M., Vancanneyt, M., Hoste, B., de Vos, P., Falsen, E., Kersters, K. \& Hinz, K.-H. (1996). Bordetella trematum sp. nov., isolated from wounds and ear infections in humans, and reassessment of Alcaligenes denitrificans Rüger and Tan 1983. Int J Syst Bacteriol 46, 849-858.

Verwey, W. F., Thiele, E. H., Sage, D. N. \& Schuchardt, L. F. (1949). A simplified liquid medium for the growth of Haemophilus pertussis. J Bacteriol 58, 127-134.

Weiss, A. A. \& Falkow, S. (1984). Genetic analysis of phase change in Bordetella pertussis. Infect Immun 43, 263-269.

Weiss, A. A., Hewlett, E. L., Myers, G. A. \& Falkow, S. (1983). Tn-5 induced mutations affecting virulence factors of Bordetella pertussis. Infect Immun 42, 33-41.

Weyant, R. S., Hollis, D. G., Weaver, R. E. \& 7 other authors (1995). Bordetella bolmesii sp. nov., a new gram-negative species associated with septicemia. J Clin Microbiol 33, 1-7.

Willems, R. J. L., van der Heide, H. G. J. \& Mooi, F. R. (1992). Characterization of a Bordetella pertussis fimbrial gene cluster which is located directly downstream of the filamentous haemagglutinin gene. Mol Microbiol 6, 2661-2671.

Zarrouk, H., Karibian, D., Bodie, S., Perry, M. B., Richards, J. C. \& Caroff, M. (1997). Structural characterization of the lipid A of three Bordetella bronchiseptica strains: variability of fatty acid substitution. J Bacteriol 179, 3756-3760.

van der Zee, A., Agterberg, C., van Agterveld, M., Peeters, M. \& Mooi, F. R. (1993). Characterization of IS1001, an insertion sequence element of Bordetella parapertussis. J Bacteriol 175, 141-147.

van der Zee, A., Groenendijk, H., Peeters, M. \& Mooi, F. R. (1996). The differentiation of Bordetella parapertussis and Bordetella bronchiseptica from humans and animals as determined by DNA polymorphism mediated by two different insertion sequence elements suggests their phylogenetic relationship. Int J Syst Bacteriol 46, 640-647.

Received 29 January 1998; accepted 26 February 1998. 